\title{
Continuous convergence and the Hahn-Banach problem
}

\section{Ronald Beattie}

\begin{abstract}
In this note, a positive solution is given to the Hahn-Banach problem for an important class of convergence vector spaces. As well, a topological vector space characterization is obtained for fully complete and $B_{p}$-complete spaces.
\end{abstract}

\section{Introduction and preliminaries}

Let $E$ be a Hausdorff locally convex topological vector space over $R$ and $L_{c}(E)$ its dual space equipped with the continuous convergence structure. In this note, we examine the relationship between the HahnBanach extension property for closed subspaces of the convergence vector space $L_{c}(E)$ and completeness conditions on $E$. In particular, every closed subspace of $L_{C}(E)$ has the extension property if and only if $E$ is fully complete. This relationship allows us to formulate the following topological vector space characterization of fully complete and $B_{r}$-complete spaces: $E$ is fully complete $\left(B_{r}\right.$-complete) if and only if every image of $E$ by a (one-to-one) continuous and nearly open mapping is complete.

Throughout, all spaces are assumed to be Hausdorff. As well, mappings are always assumed to be linear. If $E$ is a locally convex topological vector space, $L(E)$ will denote its dual space and $L_{c}(E)$ its C-dual; that is, the resulting convergence vector space when $L(E)$ carries the

Received 23 August 1977. The author thanks Dr H.-P. Butzmann for the idea behind the proof of the implication (3) $\Rightarrow(1)$ in Theorem 2.2. 
continuous convergence structure. For definitions and properties of convergence structures in general and the continuous convergence structure in particular, we refer to [1].

\section{The Hahn-Banach problem for $L_{c}(E)$}

A subspace $V$ of a convergence vector space $W$ is said to have the Hahn-Banach extension property if every continuous functional $\varphi: V \rightarrow R$ has a continuous extension $\tilde{\varphi}$ to $W$. Extension theorems in general convergence vector spaces are rather difficult to obtain (see [7]). For c-dual spaces, Proposition III.3 and Theorem III.4 of [8] show that, even for a Fréchet space $E$, it is possible to find a subspace $V$ of $L_{c}(E)$ and a continuous functional $\varphi$ on $V$ which cannot be extended to the adherence of $V$. In the light of this situation, we restrict our attention to the closed subspaces of $L_{c}(E)$.

Let $E$ and $F$ be locally convex topological vector spaces with 0 neighbourhood filters $U$ and $V$ respectively, and let $f: E \rightarrow F$ be a continuous mapping. We recall that $f$ is called open (nearly open) if the filter $f(U) \quad\{\overline{f(U)}\}$ is coarser than $U$.

LEMMA 2.1. Let $E$ and $F$ be locally convex topological vector spaces and $f: E \rightarrow F$ a continuous mapping. Then the adjoint $f^{*}: L_{C}(F) \rightarrow L_{c}(E)$ is an embedding if and only if $f$ is nearly open.

Proof. Suppose $f$ is nearly open. Then $f(E)$ is dense in $F$ and, since $f$ is continuous, $f^{*}$ is a continuous injection. $L_{c}(E)$ and $L_{c}(F)$ are locally compact convergence vector spaces ([2], Satz 5) and thus each is the inductive limit in the category of convergence spaces of its compact sets ([2], Lemma 1). Therefore, $f^{*}$ is an embedding if and only if the images under $f^{*}$ of the compact sets of $L_{c}(F)$ are precisely the compact sets of $L_{C}(E)$ contained in the image of $f^{*}$. However, the relatively compact sets of $L_{c}(E)$ and $L_{c}(F)$ are the equicontinuous subsets of $L(E)$ and $L(E)$ respectively ([4], Theorem 7 ). Thus, if $E_{E}$ and $E_{F}$ denote the collections of equicontinuous subsets of $L(E)$ and $L(F)$ respectively, $f^{*}$ is an embedding if 


$$
f^{*}\left(E_{F}\right)=E_{E} \cap f^{*}(L(F))
$$

The inclusion $f^{*}\left(E_{F}\right) \subset E_{E} \cap f^{*}(L(F))$ follows immediately from the continuity of $f^{*}$. The reverse inclusion holds if and only if $f$ is nearly open. (See for example, [5], 5.11.)

Assume, now, that $f^{*}$ is an embedding. Then $E_{E} \cap f^{*}(L(E)) \subset f^{*}\left(E_{F}\right)$ and so $f$ is nearly open.

Since the collection of polars $\left\{U^{0}: U \in U\right\}$ is cofinal in the system of compact subsets of $L_{c}(E)$, and since the continuous convergence structure agrees with the weak* topology $\sigma(L(E), E)$ on each $U^{O}$ ([4], Lemma 1), we have

$$
L_{C}(E)=\operatorname{ind}_{U \in U}\left(U^{0}, \sigma(L(E), E)\right),
$$

the inductive limit in the category of convergence spaces of the polars carrying the $\sigma(L(E), E)$ topology. A set $A \subset L(E)$ is closed in $L_{c}(E)$ or c-closed if and only if, for every $U$ in $U, A \cap U^{O}$ is $\sigma(L(E), E)$ closed. We recall that a locally convex topological vector space $E$ is fully complete if every c-closed subspace of $L(E)$ is $\sigma(L(E), E)$-closed, and $B_{r}$-complete if every c-closed and $\sigma(L(E), E)$-dense subspace of $L(E)$ coincides with $L(E)$.

THEOREM 2.2. Let $E$ be a complete locally convex topological vector space. Then the following are equivalent:

(1) E is fully complete;

(2) every image of $E$ by a continuous and nearly open mapping is complete;

(3) every closed subspace of $L_{c}(E)$ has the Hainn-Banach extension property.

Proof. We show (i) $\Rightarrow(2) \Rightarrow(3) \Rightarrow(1)$.

(1) $\Rightarrow(2)$. This follows from the open mapping theorem for fully complete spaces and the fact that quotients of fully complete spaces are 
themselves fully complete and thus complete.

$(2) \Rightarrow(3)$. Assume that $E$ satisfies (2). Let $V$ be a closed subspace of $L_{c}(E)$ and $e: V \rightarrow L_{c}(E)$ be the natural embedding. $V$ is locally compact and is itself an $L_{c}(F)$ for some locally convex topological vector space $F$ ([2], Satz 5). Then the adjoint of $e$, $e^{*}: L_{c} L_{c}(E) \rightarrow L_{c} L_{c}(F)$ is a continuous mapping. But the $c$-bidual $L_{c} L_{c}(F)$ of $F$ can be identified with the completion $\tilde{F}$ of $F$ ([3], Satz 8). Moreover, $L_{c}(F)=L_{c}(\tilde{F}) \quad([\tilde{j}]$, Satz 7$)$. Since $e=e^{* *}$, by Lemma 2.1, $e^{*}: E \rightarrow \tilde{F}$ must be nearly open. Thus $e^{*}(E)$ is dense in $\tilde{F}$. Since $E$ satisfies (2), however, $e^{*}(E)$ is complete, so that $e^{*}: L_{c} L_{c}(E) \rightarrow L_{c} L_{c}(F)$ is surjective. Thus $V=L_{c}(F)$ has the HahnBanach extension property in $L_{c}(E)$.

$(3) \Rightarrow(1)$. Assume that $L_{C}(E)$ satisfies (3). Let $V$ be a closed subspace of $L_{C}(E)$ and $x_{0} \in L(E) V$. Consider the subspace $V^{\prime}=V+R x_{0}$ of $L_{c}(E)$. Since $V$ is a closed hyperplane of $V+R x_{0}, V$ is a direct summand of $V^{\prime}$. The proof of this is the same as for locally convex topological vector spaces (see [9], p. 96, Corollary) and requires only the fact, proved in [6], that a finite dimensional vector space has only one Hausdorff convergence vector space structure. Thus $V^{\prime}=V \oplus R x_{0}$ and $V^{\prime}$ is a complete ([2], Satz 5, Korollar) and hence closed subspace of $L_{C}(E)$.

Since $V$ is a direct summand of $V^{\prime}$, the functional $\varphi$ which is 0 on $V$ and $l$ at $x_{0}$ is continuous on $V^{\prime}$. Since $L_{c}(E)$ satisfies (3), $V^{\prime}$ has the Hahn-Banach extension property, so that $\varphi$ has a continuous extention $\tilde{\varphi}$ to $L_{c}(E)$. Hence $\tilde{\varphi}$ is a continuous functional on $L_{c}(E)$ which separates $V$ and $x_{0}$. But

$$
L L_{c}(E)=L(L(E), \sigma(L(E), E))=E,
$$

so that $\tilde{\varphi}$ is a continuous functional on $(L(E), \sigma(L(E), E))$ which separates $V$ and $x_{0}$. Hence $V$ is $\sigma(L(E), E)$-closed and $E$ is fully complete. 
COROLLARY 2.3. Let $E$ be a complete locally convex topological vector space. Then the following are equivalent:

(1) $E$ is $B_{p}$-complete;

(2) every image of $E$ by a one-to-one, continuous, and nearly open mapping is complete;

(3) every c-closed and $\sigma(L(E), E)$-dense subspace of $L_{c}(E)$ has the Hahn-Banach extension property.

Proof. The proof is similar to that of Theorem 2.2 and uses the fact that a subspace $L_{c}(F)$ of $L_{c}(E)$ is $\sigma(L(E), E)$-dense in $L(E)$ if and only if the adjoint $e^{*}: E \rightarrow L_{c} L_{c}(F)$ of the embedding $e$ is one-to-one.

COROLLARY 2.4. Let $E$ be a complete locally convex topological vector space. Then the following are equivalent:

(1) $E$ is quotient complete, that is, every image of $E$ by a continuous and open mapping is complete;

(2) every $\sigma(L(E), E)$-closed subspace of $L_{c}(E)$ has the HahnBanach extension property.

Proof. The proof is similar to that of Theorem 2.2 and uses the fact that a subspace $L_{c}(E)$ of $L_{c}(E)$ is $\sigma(L(E), E)$-closed in $L(E)$ if and only if the adjoint $e^{*}: E \rightarrow L_{c} L_{c}(F)$ of $e$ is an open mapping onto its range.

In order to simplify the study of which subspaces inherit the HahnBanach extension property, we introduce the following notation. A c-dual $L_{c}(E)$ of a complete locally convex topological vector space $E$ is called an

HBI space if every closed subspace of $L_{c}(E)$ has the Hahn-Banach extension property,

HB2 space if every $c$-closed and $\sigma(L(E), E)$-dense subspace of $L_{c}(E)$ has the Hahn-Banach extension property,

HB3 space if every $\sigma(L(E), E)$-closed subspace of $L_{c}(E)$ has the 
Hahn-Banach extension property.

The equivalences given in Theorem 2.2 and Corollaries 2.3 and 2.4 together with well-known properties of locally convex topological vector spaces easily yield the following results: every closed subspace of an $\mathrm{HBl}$ space is itself an $\mathrm{HBI}$ space. Every $c$-closed and $\sigma\left(L\left(E^{\prime}\right), E\right)$-dense subspace of an HB2 space is itself an $H B 2$ space. Finally, every $\sigma(L(E), E)$-closed subspace of an HB3 space is itself an HB3 space.

On the other hand, it is unknown whether every closed subspace of an HB2 or HB3 space must itself be an HB2 or HB3 space. In fact, these questions are equivalent to the long standing problems of whether every $B_{r}$-complete space is fully complete and whether every quotient complete space is fully complete.

\section{References}

[1] Ernst Binz, Continuous convergence on $C(X)$ (Lecture Notes in Mathematics, 469 . Springer-Verlag, Berlin, Heidelberg, New York, 1975).

[2] Ernst Binz, Heinz-Peter Butzmann und Kurt Kutzler, "Über den c-Dual eines topologischen Vektorraumes", Math. 2. 127 (1972), 70-74.

[3] H.-P. Butzmann, "Über die c-Reflexivität von $C_{c}(X)$ ", Comment. Math. Helv. 47 (1972), 92-101.

[4] C.H. Cook and H.R. Fischer, "On equicontinuity and continuous convergence", Math. Arn. 159 (1965), 94-104.

[5] John Horváth, "Locally convex spaces", Stomer School on Topological Vector Spaces, 41-83 (Lecture Notes in Mathematics, 331. Springer-Verlag, Berlin, Heidelberg, New York, 1973).

[6] Kurt Kutzler, "Eine Bemerkung über endlichdimensionale, separierte, limitierte Vektorräume", Arch. Math. (Basel) 20 (1969), 165-168.

[7] Terrence S. McDermott, "The Hahn-Banach theorem in convergence vector spaces - a brief survey", Proceedings of the Special Session on Convergence Structures, 148-154 (Department of Mathematics, University of Nevada, Reno, 1976). 
[8] Bernd Müller, "Locally precompact convergence vector spaces", Proceedings of the Special session on convergence Strustures, 155-172 (Department of Mathematics, University of Nevada, Reno, 1976).

[9] A.P. Robertson and Wendy Robertson, Topological vector spaces, First edition, reprinted (Cambridge Tracts in Mathematics and Mathematical Physics, 53. Carntrirge University Dress, Cambringe, 1966).

Lehrstuhl für Mathematik I, Uni versität Mannheim, Mannheim, jermany. 